\title{
INVESTIGACION
}

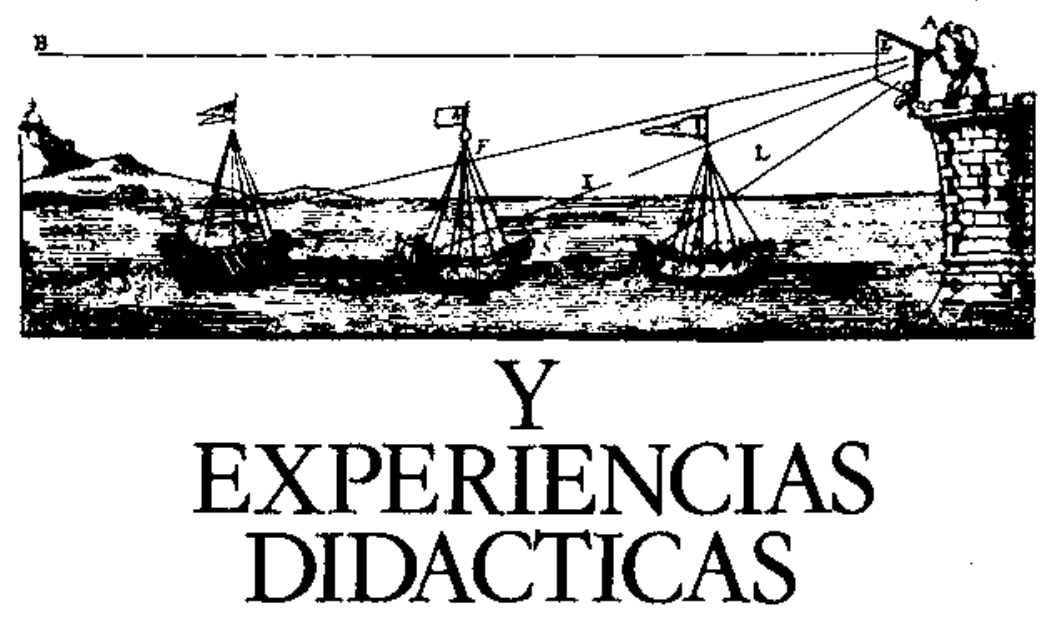

\section{CARACTERÍSTICAS DE LOS EJERCICIOS DE PRÁCTICAS DE LABORATORIO INCLUIDOS EN LOS LIBROS DE TEXTO DE CIENCIAS UTILIZADOS EN CATALUÑ̃}

TAMIR, P.' y GARCÍA ROVIRA, M. Pilar ${ }^{2}$

1. School of Education and Israel Science Teaching Center. Hebrew University. Jerusalem.

2. Departament de Didàctica de la Matemàtica i de les Ciències Experimentals. Universitat Autònoma de Barcelona. 08193 Bellaterra.

\section{SUMMARY}

The laboratory exercices in science textbooks used in grades 9 to 12 in Catalonian schools were content analyzed by three instruments: The Inquiry Level Index (ILI), the Laboratory Assessment Inventory (LAI) and the Laboratory Dimenssions Inventory (LDI). The analysis was carried out by 24 teachers under the guidance of two science educators as an assignment in an in -service course on teaching and evaluation in the laboratory. It was found that the number of laboratory exercises offered in Catalonian science texbooks is rather small compared with texbooks in countries such as the U.K. and U.S.A. Most of the exercises are of low inquiry level featuring manipulation but missing high other inquiry skills such as formulating questions and hypotheses, designing experiments, etc. The relationship between practical work and theory is hardly indicated and the potential of the laboratory as a means to enhancing concept learning and/or developing social skills are hardly being considered. There is an urgent need to reform laboratory work in Catalonian schools. 


\section{INTRODUCCIÓN}

El elemento más característico del aprendizaje de las ciencias en los centros de enseñanza es el laboratorio de ciencias. Entendemos por laboratorio de ciencias, aquel lugar especialmente equipado de un centro de enseñanza donde se dan algunas clases en las que los alumnos realizan, por sí mismos, investigaciones sobre fenómenos y organismos, y resuelven problemas utilizando diversas habilidades manuales e intelectuales.

El potencial educativo del trabajo práctico en el laboratorio es enorme (Shulman, Tamir 1973, HegartyHazel 1990). Desgraciadamente, es frecuente que los profesores no se den cuenta de este potencial, por lo que las lecciones impartidas en el laboratorio se convierten, a menudo, en unos ejercicios en los que, como en los recetarios de cocina, los estudiantes siguen una serie de instrucciones de las que sacan muy poco provecho en lo que se refiere a su aprendizaje básico (Friedler, Tamir 1984, Novak, Gowin 1984). Diversos estudios demuestran que la razón principal por la que no se alcanzan los objetivos educativos es la falta de oportunidades ofrecidas a los estudiantes para aprender los conocimientos y habilidades relacionados con esos objetivos (Comber, Keeves 1973).

Existe un método relativamente simple y económico para determinar las posibilidades de aprendizaje ofrecidas por un currículo específico. Este método es el análisis del contenido. Entendemos como tal, el análisis cuantitativo sistemático de materiales de enseñan$z a$, como libros de texto, formularios de prácticas o películas, realizado según una serie de categorías representativas de los objetivos educativos. Se han ideado y utilizado varios métodos de análisis del contenido para la evaluación de los currículos científicos. En el estudio que nos ocupa, los métodos más adecuados son los propuestos por Herron (1971), para determinar el nivel de indagación de las prácticas de laboratorio, y por Tamir y Lunetta (1978) para identificar las habilidades requeridas para la realización de un determinado trabajo práctico. Utilizando este método (ver más adelante), Herron ([971) encontró que la mayor parte de los ejercicios de laboratorio que figuraban en los libros de texto del currículo reformado de la década 1960-70 en Ios EEUt, como el PSSC oel BSCS, tenían un nivel de indagación bajo. Análogamente, mediante el Laboratory Assessment Inventory (LAI, ver más adelante), se vio que las posibilidades de utilización de una serie de habilidades como, por ejemplo, la formulación de preguntas o el diseño del control en un experimento, eran prácticamente nulas, incluso en currículos preparados especialmente para la inđagación, como los mencionados anteriormente (Tamir, Lunetta 1978, Lunetta, Tamir 1981). Gracias a estos resultados, el Israel Highschool Biology Project ha llevado a cabo, en los últimos veinte años, más de un centenar de investigaciones que proporcionan un amplio espectro de habilidades, lo que hace aumentar las posibilidades de los estudiantes de Israel para adquirirlas (Tamir 1981).

\section{OBJETIVO DE ESTE ESTUDIO}

\author{
El objetivo de este estudio es doble:
}

1. Por una parte, determinar las características de los ejercicios de laboratorio de los libros de texto de ciencias más utilizados en Cataluña, la mayoría de los cuales se utilizan también en el resto de España.

2. Por otra, demostrar que la colaboración de los profesores en la investigación educativa puede aportar información útil sobre aspectos importantes de la enseñanza de las ciencias.

\section{MÉTODO}

Se pidió a 24 licenciados que asistían a un curso denominado «Planning Teaching and Evaluation of Learning in the Science Laboratory", realizado en el Departament de Didactica de les Ciències Experimentals de la UAB, que escogieran un ejercicio de laboratorio de un libro de texto de ciencias, de los que normalmente se usan en los centros de enseñanza de Cataluña, para analizarlo desde los puntos de vista siguientes:

1. El nivel de indagación en el trabajo práctico de laboratorio (ILI, The Inquiry Level Index). Este instrumento fue diseñado por Herron (1971). El nombre ILI ha sido propuesto por el primero de los autores de este trabajo. El ILI consta de 4 niveles determinados a partir de las tareas que los estudiantes deben realizar. Nivel cero: Se les da la pregunta, el método y la respuesta. El estudiante debe seguir las instrucciones y obtener los resultados indicados en el texto. Este tipo de práctica de laboratorio se denomina también de verificación o confirmación. Nivel uno: Se da la pregunta y el método, y el estudiante tiene que hallar la respuesta. Nivel dos: Se da la pregunta y el estudiante debe encontrar un método y una respuesta. Nivel tres: Se le indica un fenómeno y tiene que formular una pregunta adecuada y encontrar un método y una respuesta a la pregunta.

2. El Laboratory Assessment Inventory (LAI, Inventario de habilidades para evaluar las activiades de laboratorio) diseñado por Tamir y Lunetta (1978). Consta de 4 categorías principales: planificación, realización, análisis y aplicación, cada una de las cuales está representađa por habilidades características, en total 28 (Tabla III). Para cada una de las habilidades el evaluador debe anotar SÍ o NO. SÍ significa que la habilidad đebe ser utilizada, NO indica que no se requiere explícitamente al estudiante para que ejercite dicha habilidad.

3. The laboratory Dimensions Inventory (LDI, Inventario de dimensiones para evaluar el trabajo práctico) ha sido diseñado, especialmente para este estudio, por el primero de los autores. Consiste en 8 dimensiones expresadas en forma de preguntas a las que hay que contestar también SÍ o NO. A continuación hacemos una breve descripción de cada dimensión. 
a. Dimensión social. ¿Los estudiantes han de trabajar individualmente o en pequeños grupos? ¿Han de realizar todos la misma investigación o, por el contrario, cada grupo investiga un aspecto diferente y luego se ponen los resultados en común? ¿Se les pide una discusión de los resultados después de la práctica? ¿Se establece alguna relación con aplicaciones sociales o tecnológicas?

b. Conocimientos previos. ¿Qué conocimientos previos se necesitan para poder realizar adecuadamente el trabajo de laboratorio? ¿Han adquirido los estudiantes las habilidades técnicas necesarias para su realización?

c. Relación con la teoría. ¿Se considera que la teoría es básica para la investigación? ¿Es necesario encontrar una explicación a las hipótesis mediante el soporte teórico pertinente? ¿Se pide a los estudiantes que relacionen sus resultados y conclusiones con la teoría?

d. Obtención de datos. ¿Cómo se obtienen los datos? ¿Gracias a observaciones directas, mediante indicadores (por ejemplo cambio del color), utilizando aparatos de registro automáticos, o bien, mediante ordenador?

e. Complejidad de los instrumentos. ¿La complejidad de los instrumentos es adecuada a la finalidad que se persigue? Por ejemplo, ¿se utiliza un medidor electrónico de $\mathrm{pH}$ o papel indicador de $\mathrm{pH}$ para diferenciar los ácidos de las bases cuando el papel de tornasol puede sernos igualmente útil?

Tabia I

Libros de texto seleccionados, disciplina y tema de la práctica de laboratorio analizada.

N. Disciplina y tema

\begin{tabular}{|c|c|c|}
\hline 1 & Biología & $\begin{array}{l}\text { La temperatura en } \\
\text { distintos hábitats }\end{array}$ \\
\hline 2 & Biologia & Fotosintesis \\
\hline 3 & Física & Fuerzas y movimiento \\
\hline 4 & Biología & Células y microscopio \\
\hline 5 & Geología & Observación sedimentos \\
\hline 6 & Biología & $\begin{array}{l}\text { Composición química } \\
\text { de los organismos }\end{array}$ \\
\hline 7 & Física & Corrientes de convección \\
\hline 8 & Quimica & $\begin{array}{l}\text { Separación de los compo- } \\
\text { nentes de una mezcla }\end{array}$ \\
\hline 9 & Química & Identificación de ejementos \\
\hline 10 & Biología & $\begin{array}{l}\text { Observación de } \\
\text { microorganismos }\end{array}$ \\
\hline 11 & Biología & $\begin{array}{l}\text { Observación de } \\
\text { microorganismos }\end{array}$ \\
\hline 12 & Química & Solubilidad \\
\hline
\end{tabular}

22

Biología

Biología

Biología

Biología

31

Bjología

Biología

Biología

Biología

Biología

41 Biología

42 Química

Título del libro de texto

Editor

\section{Ciències Naturals lBUP \\ Ciències Naturals 1 BUP \\ Fisica y Química 2 BUP \\ Cjències Naturals IBUP \\ Àmbit 1BUP \\ Ciències Naturals IBUP}

Ciencias Naturales IBUP

Problemes i pràctiques

de Química 2BUP

Àmbit 1 BUP

Àmbit $1 \mathrm{BUP}$

¿Cómo identificar

substancias puras?

Ciències Naturales 3BUP

Investigaciones de Labora-

torio y de campo

El Cos Huma

El Cos Huma

Guia de prácticas elaborada por el profesor

Prácticas de Biología de BUP

COU y FP

Biologia COU

Biología COU

Prácticas de Biología

Nuevo curriculum

Nuevo curriculum
Ed. Santiliana (1988)

Ed. Santiłana (1988)

Ed. Anaya (1990)

Ed. Santillana (1988)

Ed. Barcanova (1985)

Ed. Teide (1988)

Ed. Anaya (1988)

Ed. Grup Promotor (1979)

Ed. Barcanova (1985)

Ed. Barcanova (1985)

Grup Recerca. ICE UAB

Ed. Anaya (1988)

CECSA (1977)

Ed. Alhambra (1990)

Ed, Alhambra (1990)

Milà, Carles

Documentos Didácticos

ICE Unjv. Salamanca

Ed. Anaya (1989)

Ed. Anaya (1989)

Cuello, J. et al. (1978)

Materiales Experientales Materiales Experientales 
f. Análisis de datos. ¿Qué tipos de análisis se piden en el texto? ¿Son adecuados? ¿Existe una alternativa mejor? ¿Se ayuda a los estudiantes a encontrar la forma más idónea de expresar, presentar y comunicar los datos?

g. Tiempo. ¿Sugiere el texto la duración que debería tener la investigación? Si se tiene en cuenta el tiempo dedicado a la investigación, ¿se considera que vale la pena realizarla? El tiempo necesario para su realización, ¿es compatible con la distribución del horario escolar? ¿Se puede plantear una utilización más efectiva del tiempo como, por ejemplo, investigar problemas de genética, mediante simulaciones con ordenador?

h. Aprendizaje de conceptos. ¿Está pensado el trabajo de laboratorio para enseñar un concepto importante? Las actividades sugeridas, $i$ ayudan a abandonar las «ideas previas" y a adoptar los conceptos científicos adecuados?

Como puede observarse es posible que se produzca un cierto solapamiento entre determinadas categorías. Por ejemplo, entre la categoría $d$, obtención de datos, y la categoría $e$, complejidad de los instrumentos. De todas formas, cada categoría proporciona una información específica.

La selección de los libros de texto y los ejercicios de laboratorio analizados la realizaron los licenciados, todos ellos profesores con experiencia en el campo de la enseñanza, según su propio criterio. De ahí que puedan ser considerados como representativos de los libros de

Tabla II

Análisis de las prácticas de laboratorio en libros de texto de ciencias de $1^{\underline{a}}$ y $2^{a}$ de BUP.

\begin{tabular}{lccccccccccc} 
Tipo & 1 & 2 & 3 & 4 & 5 & 6 & 7 & 8 & 9 & 10 & 12 \\
\hline Nivel de indagacion & 1 & 1 & 1 & 0 & 0 & 0 & 1 & 0 & 2 & 1 & 1
\end{tabular}

1. PLANIFICACIÓN*

$\begin{array}{llllllllllll}1.1 & 0 & 0 & 0 & 0 & 0 & 0 & 0 & 0 & 0 & 0 & 0 \\ 1.2 & 0 & 0 & 0 & 0 & 0 & 0 & 0 & 0 & 0 & 0 & 0 \\ 1.3 & 0 & 3 & 0 & 0 & 0 & 0 & 0 & 0 & 1 & 0 & 0 \\ 1.4 & 0 & 0 & 1 & 0 & 0 & 0 & 0 & 0 & 1 & 0 & 0 \\ 1.5 & 0 & 0 & 0 & 0 & 0 & 0 & 0 & 0 & 0 & 0 & 0\end{array}$

2. REALIZACIÓN*

$\begin{array}{llllllllllll}2.1 \mathrm{a} & 1 & 3 & 1 & 1 & 3 & 1 & 1 & 1 & 2 & 1 & 1 \\ 2.1 \mathrm{~b} & 1 & 0 & 2 & 0 & 1 & 0 & 1 & 0 & 1 & 0 & 0 \\ 2.2 & 1 & 1 & 2 & 0 & 1 & 1 & 2 & 1 & 1 & 2 & 0 \\ 2.3 & 1 & 3 & 1 & 0 & 3 & 1 & 3 & 0 & 1 & 1 & 3 \\ 2.4 & 0 & 0 & 1 & 0 & 0 & 0 & 0 & 0 & 1 & 0 & 1 \\ 2.5 & 1 & 3 & 1 & 0 & 0 & 0 & 0 & 0 & 1 & 0 & 1 \\ 2.6 & 0 & 0 & 0 & 0 & 0 & 0 & 0 & 0 & 0 & 0 & 3\end{array}$

3. ANÁLISIS*

$\begin{array}{llllllllllll}3.1 \mathrm{a} & \mathrm{I} & 0 & 1 & 0 & 0 & 0 & 1 & 0 & 1 & 0 & 1 \\ 3.1 \mathrm{~b} & 1 & 0 & 1 & 0 & 0 & 0 & 0 & 0 & 0 & 0 & 0 \\ 3 . \mathrm{Ic} & 0 & 0 & 0 & 0 & 0 & 0 & 0 & 0 & 0 & 1 & 0 \\ 3.2 & 0 & 3 & 1 & 0 & 0 & 1 & 2 & 0 & 3 & 1 & 3 \\ 3.3 & 1 & 0 & 1 & 0 & 0 & 0 & 0 & 0 & 1 & 0 & 1 \\ 3.4 & 0 & 0 & 0 & 0 & 0 & 0 & 0 & 0 & 1 & 0 & 1 \\ 3.5 & 0 & 0 & 0 & 0 & 0 & 0 & 0 & 0 & 2 & 0 & 2 \\ 3.6 & 0 & 3 & 2 & 0 & 0 & 1 & 0 & 0 & 1 & 0 & 1 \\ 3.7 & 0 & 0 & 0 & 0 & 0 & 0 & 2 & 0 & 0 & 0 & 0\end{array}$

4. APLICACION*

$\begin{array}{llllllllllll}4.1 & 1 & 0 & 0 & 0 & 0 & 0 & 1 & 1 & 3 & 0 & 3 \\ 4.2 & 0 & 0 & 0 & 0 & 0 & 0 & 0 & 1 & 2 & 0 & 2 \\ 4.3 & 0 & 0 & 0 & 0 & 0 & 1 & 0 & 1 & 0 & 0 & 0 \\ 4.4 & 0 & 0 & 0 & 0 & 0 & 0 & 1 & 1 & 0 & 0 & 0\end{array}$


tex to actualmente utilizados en la enseñanza secundaria en Cataluña (Tabla I). Todos los análisis fueron comprobados por los autores de este artículo y se hicieron las correcciones necesarias.

EI examen de los libros de texto seleccionados puso de manifiesto que representaban los tres tipos de poblaciones siguientes: a) $1^{2}$ y $2^{\circ}$ de BUP, ciencias obligatorias; b) $3^{\circ}$ de BUP, ciencias electivas; y c) COU opción de ciencias para el ingreso en la universidad, materias obligatorias u optativas en función de la opción. Para reforzar la generalización de los resultados, se sometió a un análisis de contenido, de todas sus prácticas de laboratorio, un conocido libro de texto del cual existen versiones utilizadas por cada una de las tres poblaciones mencionadas anteriormente (Ciències Naturals ir BUP, Ed. Santillana 1988; Ciències Naturals 3r BUP, Ed. Santillana 1989; Biología COU, Ed. Santillana 1985). Este análisis fue realizado por los autores de este artículo y sus resultados se dan por separado.

\section{RESULTADOS}

Las Tablas II, III y IV muestran los resultados del análisis cuantitativo realizado por los licenciados utilizando el método del nivel de indagación y el LAI. Las

Tabla III

Análisis de las prácticas de laboratorio en libros de texto de ciencias de $3^{\circ}$ de BUP.

Tipo

21

$1 \quad 1 \quad 1$

Nivel de indagación

1

I. PLANIFICACIÓN. El alumno:

1.1 Formula una pregunta

1.2 Predice resultados experimentales

1.3 Formula hipotesis

1.4 Diseña observaciones/procedimientos

1.5 Diseña un experimento

$\begin{array}{llll}0 & 0 & 0 & 0 \\ 0 & 0 & 0 & 0 \\ 0 & 0 & 0 & 0 \\ 0 & 0 & 0 & 0 \\ 0 & 0 & 0 & 0\end{array}$

2. REALIZACION. EI alumno:

2.1a Realiza observaciones

2.1b Realiza medidas

2.2 Utiliza aparatos

2.3 Anota resultados

2.4 Hace calculos numericos

2.5 Explica procedimientos

2.6 Trabaja según su propio disẹno

$\begin{array}{llll}2 & 1 & 3 & 2 \\ 2 & 3 & 3 & 0 \\ 3 & 1 & 3 & 2 \\ 2 & 1 & 3 & 1 \\ 0 & 1 & 0 & 1 \\ 1 & 3 & 0 & 0 \\ 0 & 0 & 0 & 0\end{array}$

3. ANÁLISIS. El alumno:

3.1 a Representa los datos en una tabia

3.1b Representa los resultados gráfjcamente

3.Ic Realiza dibujos basados en sus propias observaciones

3.2 Extrae interrelaciones y/o conclusiones

3.3 Determina la exactitud de los resultados experimentales

3.4 Define las limitaciones y los supuestos inherentes al experimento

3.5 Formula generalizaciones/propone modetos

3.6 Explica interrelaciones

3.7 Formula nuevas preguntas

4. APLICACIÓN. El alumno:

4.1 Hace predicciones basadas en los resultados experimentales

4.2 Formula hipótesis basadas en los resultados experiemntales

4.3 Aplica las técnicas experimentales a un problema nuevo

4.4 Aplica los resultados experimentales a un nuevo contexto

$\begin{array}{llll}2 & 1 & 2 & 0 \\ 0 & 1 & 0 & 0 \\ & & & \\ 0 & 0 & 0 & 2 \\ 2 & 3 & 3 & 2 \\ & & & \\ 0 & 2 & 0 & 0 \\ & & & \\ 1 & 0 & 0 & 0 \\ 0 & 0 & 0 & 0 \\ 1 & 3 & 0 & 1 \\ 0 & 0 & 0 & 0\end{array}$

$0=$ ausente $1=$ aparece ana vez $2=$ aparece dos veces $3=$ aparece tres veces o más $3^{2}$ de BUP $=$ Ciencias optativas. 
Tabla IV

Análisis de las prácticas de laboratorio en libros de texto de Biología de COU.

\begin{tabular}{cccccc} 
Tipo & 31 & 32 & 33 & 34 & 35 \\
\hline Nivel de indagacion & 1 & 1 & 1 & 1 & 1 \\
\hline 1. PLANIFICACION* & & & & & \\
1.1 & 0 & 0 & 0 & 0 & 0 \\
1.2 & 0 & 0 & 0 & 0 & 0 \\
1.3 & 0 & 0 & 0 & 0 & 0 \\
1.4 & 0 & 0 & 0 & 0 & 0 \\
1.5 & 0 & 0 & 0 & 0 & 0 \\
\hline 2. REALIZACION* & & & & & \\
$2.1 \mathrm{a}$ & 3 & 3 & 2 & 1 & 1 \\
$2.1 \mathrm{~b}$ & 3 & 0 & 2 & 1 & 1 \\
2.2 & 3 & 0 & 2 & 1 & 1 \\
2.3 & 3 & 3 & 3 & 1 & 1 \\
2.4 & 3 & 0 & 1 & 0 & 0 \\
2.5 & 0 & 0 & 2 & 0 & 0 \\
2.6 & 0 & 0 & 0 & 0 & 0 \\
\hline
\end{tabular}

3. ANÁLISIS*

$\begin{array}{llllll}3.1 \mathrm{a} & 3 & 1 & 0 & 1 & 1 \\ 3 . \mathrm{b} & 2 & 0 & 0 & 0 & 0 \\ 3.1 \mathrm{c} & 3 & 0 & 0 & 0 & 0 \\ 3.2 & 3 & 3 & 2 & 1 & 1 \\ 3.3 & 1 & 0 & 0 & 0 & 0 \\ 3.4 & 1 & 0 & 1 & 0 & 0 \\ 3.5 & 1 & 0 & 0 & 0 & 0 \\ 3.6 & 3 & 3 & 1 & 0 & 0 \\ 3.7 & 0 & 0 & 0 & 0 & 0\end{array}$

4. APLICACIÓN*

4.1

4.2

4.3

4.4

I 0

30

000

$\begin{array}{lll}0 & 1 & 1 \\ 0 & 0 & 0 \\ 0 & 0 & 0 \\ 0 & 0 & 0\end{array}$

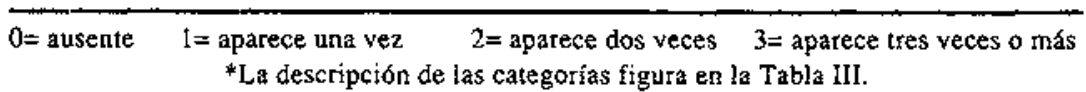

Tablas V y VI recogen, respectivamente, los resultados del análisis de los contenidos de "Ciències Naturals» y «Biologia» realizado por los autores del artículo.

Independientemente del nivel escolar y deI currículo utilizado, se ve muy claramente que es difficil poder desarrollar las habilidades de planificación o aplicación mediante los ejercicios prácticos de laboratorio que aparecen en los textos más utilizados publicados hasta ahora. Los profesores que deseen desarrollar estas habi* lidades en sus alumnos deben elaborar sus propios ejercicios de láboratorio. En cuanto a las habilidades analíticas, sólo algunas de ellas están adecuadamente representadas: 3.2 (relacionar, sacar conclusiones), 3.6 (explicar las relaciones) $\mathrm{y}$, en las prácticas de COU
(Tabla IV), 3.1 (transformación de resultados).

Dos de los licenciados que asistían al curso escogieron trabajos prácticos de laboratorio sacados del material experimental del nuevo currículo, distribuido por la administración educativa, uno de elios sobre fermenta. ción de la levadura y el otro sobre el agua clorada (Tabla 1). Nuestro análisis de contenidos demostró que estos ejercicios no son diferentes del resto: no hay referencia alguna a las habilidades de planificación, sólo una referencia a aplicación y únicamente se utilizan 3 de las 9 habilidades de análisis. Si el resto de los ejercicios de laboratorio del nuevo currículo es similar a los dos analizados aquí, es difícil que se puedan producir mejoras en la enseñanza impartida en el laboratorio. 
Tabla V

Resultados del análisis de contenidos de las prácticas de laboratorio de un libro de texto de Biología de COU (1985).

\begin{tabular}{|c|c|c|c|c|c|c|c|}
\hline Tipo & $\mathrm{N}=8$ & Céluia & Organismos & Genética & $\begin{array}{c}\text { Ecología } \\
y \\
\text { Evolución } \\
N=2\end{array}$ & Etología & Inmunología \\
\hline Nivel de indagación & $\begin{array}{l}7=0 \\
I=1\end{array}$ & $\begin{array}{l}4=0 \\
7=1\end{array}$ & $\begin{array}{l}4=0 \\
1=1\end{array}$ & $\begin{array}{l}2=0 \\
2=2\end{array}$ & $\begin{array}{l}1=0 \\
1=I\end{array}$ & $J=1$ & $I=1$ \\
\hline
\end{tabular}

1. PLANIFICACION*

$\begin{array}{llllllll}1.1 & 0 & 0 & 0 & 0 & 0 & 0 & 0 \\ 1.2 & 0 & 0 & 0 & 0 & 0 & 0 & 0 \\ 1.3 & 0 & 0 & 0 & 0 & 0 & 0 & 0 \\ 1.4 & 0 & 0 & 0 & 0 & 0 & 0 & 0 \\ 1.5 & 0 & 0 & 0 & 0 & 0 & 0 & 0\end{array}$

\begin{tabular}{cccccccc}
\hline 2. REALIZACIÓN* & & & & & & 3 & 1 \\
$2.1 \mathrm{a}$ & 17 & 26 & 13 & 10 & 6 & 0 & 0 \\
$2.1 \mathrm{~b}$ & 15 & 16 & 3 & 3 & 6 & 1 & 3 \\
2.2 & 15 & 20 & 10 & 6 & 6 & 0 & 0 \\
2.3 & 10 & 17 & 1 & 0 & 6 & 0 & 1 \\
2.4 & 0 & 0 & 1 & 6 & 0 & 0 & 0 \\
2.5 & 0 & 0 & 0 & 0 & 0 & 0 & 0 \\
2.6 & 0 & 0 & 0 & 0 & 0 & & 0
\end{tabular}

3. ANÁLISIS*

$\begin{array}{llllllll}3.1 \mathrm{a} & 0 & 0 & 0 & 0 & 0 & 0 & 0 \\ 3.1 \mathrm{~b} & 0 & 3 & 0 & 0 & 0 & 0 & 0 \\ 3.1 \mathrm{c} & 1 & 1 & 4 & 0 & 0 & 0 & 1 \\ 3.2 & 3 & 14 & 3 & 10 & 6 & 0 & 0 \\ 3.3 & 0 & 0 & 0 & 0 & 0 & 0 & 0 \\ 3.4 & 0 & 0 & 0 & 0 & 0 & 0 & 0 \\ 3.5 & 0 & 2 & 0 & 0 & 0 & 0 & 0 \\ 3.6 & 1 & 8 & 2 & 2 & 0 & 0 & 0 \\ 3.7 & 0 & 0 & 0 & 0 & 0 & 0 & 0\end{array}$

4. APLICACION*

$\begin{array}{llllllll}4.1 & 0 & 0 & 0 & 0 & 0 & 0 & 0 \\ 4.2 & 0 & 0 & 1 & 0 & 0 & 0 & 0 \\ 4.3 & 2 & 0 & 0 & 0 & 0 & 0 & 0 \\ 4.4 & 0 & 0 & 0 & 0 & 0 & 0 & 0\end{array}$

* La descripción de las categorías figura en la Tabla HII.

\section{DISCUSIÓN}

La enseñanza de las ciencias en España está a punto de cambiar; con la implantación del nuevo currículo propuesto en la Reforma se pasará de una orientación tradicional de tipo factual a dar mayor importancia a los conceptos y a los procedimientos. Sin embargo, tal como ocurre en otros países, el proceso de cambio puede ser lento. La experiencia de los países donde se produjeron reformas similares del currículo de ciencias ha demostrado que los tres factores decisivos para que se pueda aplicar con éxito el cambio en la enseñanza escolar son: el profesor, los materiales de estudio y el tipo de pruebas utilizadas para evaluar los progresos de los estudiantes.
Este trabajo se centra en la naturaleza de los materiales del currículo y en su adecuación al aprendizaje del estudiante en el laboratorio. Las dimensiones que se han seleccionado para este análisis son las que se asocian habitualmente con la contribución potencial del laboratorio al aprendizaje de las ciencias. Dos de estas dimensiones, el nivel de indagación y la utilización de habilidades de indagación específicas, proporcionaron datos cuantitativos que se han obtenido mediante la aplicación de los instrumentos antes citados (Nivel de indagación y $\mathrm{LAI}$ ) a los textos seleccionados. Las otras dimensiones consideradas casi no aparecen en los materiales analizados. 
Tabla VI

Resilltados del análisis de contenido de las prácticas de laboratorio de los libros de texto Ciències Naturals 1r de BUP (1988)

y 3r de BUP (1989).

\begin{tabular}{|c|c|c|c|c|c|c|c|c|c|c|}
\hline Tema & Geologia & Célula & $\begin{array}{l}\text { Botá- } \\
\text { nica }\end{array}$ & Zoologia & $\begin{array}{l}\text { Genética } \\
\text { Evolución }\end{array}$ & Ecología & Geología & $\begin{array}{l}\text { Bioquímica } \\
\text { Céłula }\end{array}$ & $\begin{array}{l}\text { Cuerpo } \\
\text { Humano }\end{array}$ & $\begin{array}{l}\text { Botá- } \\
\text { nica }\end{array}$ \\
\hline Currículo & $\begin{array}{l}\text { 1BUP } \\
\mathrm{N}=5\end{array}$ & $\begin{array}{l}1 \mathrm{BUP} \\
\mathrm{N}=4\end{array}$ & $\begin{array}{l}1 \mathrm{BUP} \\
\mathrm{N}=2\end{array}$ & $\begin{array}{l}1 \mathrm{BUP} \\
\mathrm{N}=3\end{array}$ & $\begin{array}{l}\text { 1BUP } \\
\mathrm{N}=3\end{array}$ & $\begin{array}{l}\text { 1BUP } \\
\mathrm{N}=4\end{array}$ & $\begin{array}{c}3 \mathrm{BUUP} \\
\mathrm{N}=6\end{array}$ & $\begin{array}{l}\text { 3BUP } \\
\mathrm{N}=3\end{array}$ & $\begin{array}{l}3 \mathrm{BUP} \\
\mathrm{N}=13\end{array}$ & $\begin{array}{l}3 B U P \\
N=4\end{array}$ \\
\hline \multirow[t]{2}{*}{ Nivel de indagación } & $2=0$ & $3=0$ & $1=0$ & $2=0$ & $1=0$ & & $4=0$ & $3=0$ & $6=0$ & \\
\hline & $3=1$ & $\mathrm{I}=1$ & $I=1$ & $\mathrm{I}=\mathrm{I}$ & $2=1$ & $4=1$ & $2=1$ & & $7=1$ & $4=1$ \\
\hline
\end{tabular}

1. PLANIFICACIÓN*

\begin{tabular}{llllllllllll}
1.1 & 0 & 0 & 0 & 0 & 0 & 0 & 0 & 0 & 0 & 0 \\
1.2 & 0 & 0 & 0 & 0 & 0 & 0 & 0 & 0 & 0 & 0 \\
1.3 & 0 & 0 & 0 & 0 & 0 & 0 & 0 & 0 & 0 & 0 \\
1.4 & 0 & 0 & 0 & 0 & 0 & 0 & 0 & 0 & 0 & 0 \\
1.5 & 0 & 0 & 0 & 0 & 0 & 0 & 0 & 0 & 0 & 0 \\
\hline
\end{tabular}

2. REALIZACIÓN

$\begin{array}{llllllrrrrrr}2.1 \mathbf{a} & 7 & 8 & 2 & 7 & 4 & 9 & 11 & 7 & 35 & 10 \\ 2.1 \mathbf{b} & 5 & 1 & 3 & 0 & 1 & 9 & 3 & 6 & 9 & 3 & 4 \\ 2.2 & 8 & 4 & 2 & 3 & 2 & 7 & 10 & 3 & 18 & 4 \\ 2.3 & 9 & 4 & 1 & 1 & 2 & 12 & 1 & 1 & 14 & 3 \\ 2.4 & 2 & 2 & 1 & 0 & 0 & 3 & 1 & 0 & 1 & 0 \\ 2.5 & 0 & 0 & 0 & 1 & 0 & 0 & 0 & 0 & 0 & 0 \\ 2.6 & 0 & 0 & 0 & 0 & 0 & 0 & 0 & 0 & 0 & 0\end{array}$

3. ANÁLISIS

$\begin{array}{lllllllllll}3.1 \mathrm{a} & 0 & 0 & 0 & 0 & 0 & 5 & 0 & 0 & 1 & 0 \\ 3.1 \mathrm{~b} & 0 & 0 & 0 & 0 & 1 & 1 & 0 & 0 & 0 & 0 \\ 3.1 \mathrm{c} & 1 & 0 & 1 & 3 & 1 & 1 & 0 & 3 & 20 & 1 \\ 3.2 & 2 & 2 & 0 & 0 & 3 & 7 & 0 & 3 & 22 & 3 \\ 3.3 & 0 & 0 & 0 & 0 & 0 & 0 & 0 & 0 & 0 & 0 \\ 3.4 & 0 & 0 & 0 & 0 & 0 & 1 & 0 & 0 & 0 & 0 \\ 3.5 & 0 & 0 & 0 & 0 & 0 & 0 & 0 & 0 & 1 & 0 \\ 3.6 & 3 & 2 & 0 & 2 & 3 & 3 & 0 & 0 & 13 & 1 \\ 3.7 & 0 & 0 & 0 & 0 & 0 & 0 & 0 & 0 & 0 & 0\end{array}$

4. APLICACION

$\begin{array}{lllllllllll}4.1 & 0 & 0 & 0 & 0 & 0 & 0 & 0 & 0 & 1 & 0 \\ 4.2 & 2 & 1 & 0 & 0 & 0 & 0 & 0 & 0 & 2 & 0 \\ 4.3 & 0 & 0 & 0 & 0 & 1 & 0 & 0 & 0 & 0 & 0 \\ 4.4 & 2 & 0 & 1 & 0 & 0 & 0 & 0 & 0 & 2 & 0\end{array}$

* La descripción de las categorías figura en la Tabila III.

Examinaremos cada dimensión por separado y, después de hacer un resumen, sacaremos las conclusiones pertinentes. Queremos resaltar que el análisis realizado se refiere a los libros de texto escritos para el currículo antiguo, si bien algunos de ellos han sido publicados en 1990. Sin embargo, nuestras conclusiones tienen importantes consecuencias de cara al diseño de los materiales para los nuevos currículos que se utílizarán en un futuro próximo.
Conocimientos previos. Si consideramos únicamente las prácticas de laboratorio de verificación, los conocimientos previos no son, normalmente, una cuestión problemática, ya que los estudiantes realizan unas actividades para verificar fenómenos y conceptos que ya han aprendido. Este hecho puede explicar la costumbre según la cual los manuales de laboratorio normalmente no especifican los conocimientos que se consideran necesarios para poder llevar a cabo determinadas investigaciones, y que se dan 
por adquiridos. Los resultados de este trabajo demuestran que, en nuestra muestra, tampoco hay referencia alguna a los conocimientos previos. Creemos que cuanto más se utilicen los laboratorios orientados a la «indagación,» mayor será la necesidad de concretar los conocimientos previos requeridos si lo que pretendemos es proporcionar unas oportunidades razonables para que se puedarn solucionar con éxito los problemas. Esta concreción, tanto en el texto del estudiante como en la guía del profesor, facilitará que los profesores mantengan discusiones previas a los trabajos de laboratorio y que, en consecuencia, los trabajos prácticos de este tipo adquieran su pleno sentido.

La dimensión social. Al igual que los conocimientos previos, la dimensión social falta normalmente en los manuales de laboratorio. Entre todos los ejercicios de Iaboratorio analizados, sólo hemos encontrado uno que diera instrucciones concretas en relación al ámbito social: se trata de una actividad al aire libre, en el capítulo 27 de las «Ciències Naturals» para $1^{\circ}$ de BUP, en la que se dan unas indicaciones específicas para formar grupos en la clase, que trabajarán en temas diferentes, para luego poner en común los resultados y examinarios conjuntamente en la clase. En todos Ios ejercicios restantes se deja que el profesor decida la formación de grupos, la asignación de las tareas a los distintos grupos y la forma de interacción con los estudiantes. Ahora bien, los profesores con experiencia, saben perfectamente que existen determinadas opciones que funcionan mejor que otras para ciertas investigaciones de laboratorio. Estas opciones podrían incluirse en el texto del estudiante o bien en la guía del profesor. Los resultados proporcionados por investigaciones recientes sobre aprendizaje cooperativo pueden proporcionar indicaciones muy útiles al respecto.

Obtención y análisis de datos. Los detalles sobre la obtención y análisis de datos relativos a lo que se espera que hagan los estudiantes están bien representados en los resultados del LAI.

Complejidad de los instrumentos. La finalidad principal de esta categoría es determinar el grado de adecuación entre los instrumentos y el fin para el que son utilizados. En todos los ejercicios analizados, los instrumentos de observación y medida son sencillos y representativos de los que normalmente se utilizan en los centros de secundaria: microscopios, termómetros, balanzas, etc. No se citan ni los ordenadores ni instrumentos más complejos como los medidores electrónicos de $\mathrm{pH}$, espectrofotómetros u otros instrumentos parecidos.

Aprendizaje de conceptos. En ninguno de los ejercicios prácticos de laboratorio hemos encontrado intento alguno de desartollar un determinado concepto cientificoutilizando la indagación sistemática đirigida.

Nivel de indagación. Todos los ejercicios de laboratorio analizados, excepto uno, son del nivel de indagación 00 1. En las raras ocasiones en que se pide a los estudiantes que indiquen cómo podrían encontrar algo, el nivel de planificación requerido es muy bajo. Por ejemplo, al estudiar el movimiento de descenso de una bola por un plano inclinado, el estudiante debe decidir en qué puntos de la trayectoria colocará la bola en cada una de las distintas observaciones.

Procedimientos y habilidades de laboratorio. Los resultados de los análisis del LAI concuerdan totalmente con tos hallados en el nivel de indagación. Las habilidades de realización, especialmente las cuatro básicas: observación, obtención de medidas, manipulación de aparatos y registro de resultados son mucho más numerosas que Ias restantes habilidades. Las habilidades de planificación como las correspondientes a formulación de preguntas de investigación, predicción, formulación de hipótesis, diseño de observaciones y experimentos, así como sus habilidades asociadas, tales como trabajar según el propio điseño y aplicar los resultados a una situación distinta, son prácticamente inexistentes. Puesto que la posibilidad de desarrollar este tipo de habilidades intelectuales se considera como una finalidad primordial del Iaboratorio de prácticas escolar, debemos concluir que los ejercicios de laboratorio existentes en los libros de texto actuales, algunos de ellos publicados hace muy poco, fallan por lo que se refiere a su finalidad educativa. Si no se produce un cambio cualitativo importante, los profesores, que necesitarán en gran medida toda la ayuda y orientaciones posibles para adaptarse al nuevo currículo, carecerán de la asistencia que podrían obtener si los ejercicios de laboratorio disponibles estuvieran más orientados hacia la indagación que los que se incluyen en los libros de tex to actuales. En relación con la formación inicial y permanente de los profesores que deberán impartir los nuevos currículos, hasta que se publiquen más ejercicios de laboratorio orientados a desarrollar la indagación, se debería insistir en la necesidad de realizar cursos de reciclaje y perfeccionamiento con la finalidad de transformar los laboratorios de bajo nivel de indagación en otros de nivel más elevado, en los que se dieran las oportunidades adecuadas para desarrollar todas las habilidades y procedimientos inherentes al trabajo experimental. Un verdadero reto para los que elaboran el nuevo currículo es el de incorporar estos ejercicios de laboratorio en sus nuevos libros de texto.

\section{CONCLUSIONES}

El número de ejercicios prácticos de laboratorio contenidos en muchos de los libros de texto analizados es muy bajo, comparado con manuales parecidos utilizados en países como Gran Bretaña y EEUU.

La mayor parte de los ejercicios analizados se caracteriza porque, para su realización, se requiere un nivel de indagación bajo. Gran parte del tiempo pasado en el laboratorio se utiliza en tareas de observación, medida, manipulación de aparatos y descripción de resultados. Este tipo de trabajo, en los laboratorios de investigación, lo realizan a menudo los técnicos de laboratorio. Para realizar los ejercicios propuestos es raro que se requieran habilidades de indagación superiores, como las correspondientes a formulación de preguntas e hipótesis, diseño de experimentos, etc. Apenas se indica la relación entre trabajo práctico y teoría y casi no se tiene 
en cuenta el potencial del trabajo de laboratorio para poner de manifiesto el aprendizaje de conceptos y/o desarrollar habilidades sociales como las recomendadas por los partidarios del aprendizaje cooperativo.

Esta deplorable situación priva a los estudiantes y profesores de Cataluña de la ayuda, potencialmente importante, que podrían of recer los materiales de aprendizaje, si éstos hubieran sido diseñados pensando en las oportunidades que deberían proporcionar para aprender los conceptos básicos y para desarrollar las habilidades procedimentales de tipo no sólo manipulativo sino también intelectual inherentes a la experimentación científica. Sin este tipo de materiales, el nuevo currículo de ciencias que está en vías de implantación, tiene pocas posibilidades de alcanzar determinados objetivos que consideramos componentes esenciales de la capacidad cientiffica.

\section{REFERENCIAS BIBLIOGRÁFICAS}

COMBER, L.E. y KEEVES, J.E.,1973. Science education in nineteen countries. (Almquist: Stockholm).

FRIEDLER, X. y TAMIR, P.,1984. Teaching and learning in high school laboratory classes in Israel, Research in Science Education, 15, pp. 89-96.

HEGARTZ-HAZEL, E. (ed.), 1990. The student laboratoryand the science curriculo. (Rutledge: London).

HERRON, M.D., 1971. The nature of scientific inquiry, School Review, 79, pp. 141-212.

LUNETTA, V.N. y TAMIR, P, 1981. Inquiry related tasks in science laboratory handbooks, Science Education, 65, pp. $477-484$

\section{NOTA}

Este estudio se realizó durante la estancia del primero de los autores de este artículo como profesor visitante en la Universitat Autònoma de Barcelona.

\section{AGRADECIMIENTOS}

Los autores agradecen la colaboración de los siguientes licenciados en la recogida de datos: Alcaraz, A.; Armengol, M. C.; Cabello, M.; Camara, J.; Carbonell, J.; Carne, G.; Correig, T.; Graells, C.; Grau R.; González, E.; Guix, M. M.; Lope, S.; Maune, A.; Maymó, S.; Menoyo, M. P.; Milà, C.; Picado, C.; Rayo, H.; Roca, M.; Rubio, M. L.; Serra, N.; Varela, X.; Ventura, M. T. y Vilar, X.

NOVAK, J.D. y GOWIN, D.B., 1984. Learning how to learn. (Cambridge University Press: New York). Aprendiendo a aprender, 1988 (Martínez-Roca: Barcelona).

SHULMAN, L.S. y TAMIR, P., 1973. Research on teaching in the natural sciences, en R.M.W. Travers (ed.) Second handbook of research on teaching. (Rand Mcdally: Chicago, pp. 1098-1140).

TAMIR, P. y LUNETTA, V.I.,1978. An analysis of Iaboratory activities in the BSCS Yellow Version, The American Biology Teacher, 40, pp. 353-357.

TAMIR, P.,1981. What can we learn from content analysis of inguiry oriented laboratory investigations, Biology Teacher Bulletin, 18, pp. 37-41 (en hebreo). 